\title{
Impact of a Screening Program in the Diagnosis, Surgical Treatment and Management of Breast Cancer
}

Inaki Lete ${ }^{1,2 *}$, Mirian Aguiar', Arguiñe Andonegui ${ }^{1}$, Nagore Zeberio' ${ }^{1}$, María Cuadra ${ }^{1}$, José Luis Mendizabal ${ }^{1,2}$ and Javier Gorostiaga ${ }^{1,2}$

${ }^{1}$ Gynecological Cancer Unit, Unit for the Clinical Management in Obstetrics and Gynecology, Araba University Hospital, Vitoria, Spain

${ }^{2}$ Faculty of Medicine, Department of Obstetrics and Gynecology, University of the Basque Country, Vitoria, Spain

\begin{abstract}
Introduction: The effects of early detection on treatment need to be evaluated. The objective of this study was to assess the impact of the Basque breast cancer early detection program (BCEDP) on breast cancer surgery in our hospital.

Materials and Methods: A retrospective study was conducted of women who underwent surgery for breast cancer between 1 January 2015 and 31 December 2016. Participants were classified into: Group A, those referred from the screening program after routine mammography; or group $B$, those referred from primary care or other gynecological clinics within our health region.

Results: In the study period, 630 women were diagnosed with and treated for breast cancer. Their mean age was 60.2 years (standard deviation 5.4). Out of these, 220 (35\%) referred from the BCEDP formed group A, the other 410 women $(65 \%)$ forming group B. Invasive cancer was diagnosed in 185 women in group A (84\%) and in $370(90.2 \%)$ in group $B(p=0.02)$. Regarding treatment, mastectomies were carried out in 47 women $(21.4 \%)$ in Group A and $225(54.9 \%)$ in group B ( $p<0.001)$; axillary lymphadenectomies were performed in 31 women $(14.1 \%)$ in Group $A$ and $83(20.2 \%)$ in Group $B,(p=0.02)$; and adjuvant chemotherapy was given in 66 women $(30 \%)$ in Group A and $148(36.1 \%)$ in Group B $(p<0.001)$.
\end{abstract}

Conclusion: In our setting, breast cancer patients referred through the BCEDP have earlier stage cancer, can be expected to benefit from less radical surgery, have lower proportion of axillary lymph node involvement and require less adjuvant chemotherapy.

Keywords: Breast cancer; Screening program; Conservative surgery; Chemotherapy

\section{Introduction}

The first screening programs for breast cancer were launched in the late nineteen-seventies based on regular mammography's [1]. These were founded on the assumption that it would be possible to reduce both morbidity and mortality associated with this condition [2]. In fact, it is considered that screening programs should facilitate the diagnosis of earlier stages of invasive ductal carcinoma (IDC) and more stages of ductal carcinoma in situ (DCIS) [3]. This earlier diagnosis should be associated with lower rates of mastectomy, radiotherapy and/ chemotherapy and with a lower rate of associated mortality [4].

Although numerous studies have quantified a reduction in breast cancer mortality in relation to screening programs, the effectiveness of screening for reducing mortality is still under discussion. While some studies have estimated that breast cancer screening programs achieve a $10 \%$ reduction in mortality after 13 years of follow up $[5,6]$ other authors estimated a rate of $20 \%$ [7], and a systematic review of European observational studies found a reduction of 31\% [8]. Despite the apparent controversy concerning the impact of screening programs on breast cancer-associated mortality rates, such programs are undoubtedly important for reducing the degree of aggressiveness required in surgery [9].

To assess the impact of the breast cancer screening program in the Basque Country on the breast cancer-related surgical procedures performed, we carried out a retrospective review analyzing the type of interventions and the stage of the disease as a function of whether patients had been diagnosed within the screening program.

\section{Material and Methods}

\section{Objective}

To assess the impact of the current breast cancer screening programme in the Basque Country on the treatment of our patients. In our health region, the Basque breast cancer early detection programme (BCEDP), based on biennial mammograms, focusses on women aged between 50 and 69 years old. If women have a family history of breast cancer, however, they are invited to join the programme with the same mammogram interval from 40 years of age. The rate of participation in this programme is about $80 \%$.

\section{Study design}

A retrospective study was conducted of women who underwent surgery in our hospital for breast cancer between 1 January 2015 and 31 December 2016.

\section{Inclusion criteria}

We included women of all ages, with primary breast carcinoma (i.e., excluding patients with metastatic disease) that had an indication

*Corresponding author: Inaki Lete, Gynecological Cancer Unit, Unit for the Clinical Management in Obstetrics and Gynecology, Araba University Hospital, Vitoria, Spain, Tel: +34 945007204; Fax: +34 945007901; E-mail: luisignacio.letelasa@osakidetza.net

Received May 16, 2018; Accepted July 11, 2018; Published July 13, 2018

Citation: Lete I, Aguiar M, Andonegui A, Zeberio N, Cuadra M et al. (2018) Impact of a Screening Program in the Diagnosis, Surgical Treatment and Management of Breast Cancer. J Cancer Sci Ther 10: 152-150. doi: 10.4172/1948-5956.1000537

Copyright: @ 2018 Lete I, et al. This is an open-access article distributed under the terms of the Creative Commons Attribution License, which permits unrestricted use, distribution, and reproduction in any medium, provided the original author and source are credited. 
for surgical treatment and underwent surgery in our hospital. Women were excluded if they had inoperable breast cancer or if, due to their health status, it was decided to administer hormone therapy or palliative chemotherapy. Patients that have locally advanced breast cancer at diagnosis or in whom the tumour-to-breast volume ratio precludes breast-conserving surgery are usually treated by the medical oncology department of our hospital using six cycles of neoadjuvant chemotherapy.

In our hospital, we follow the protocol of the Spanish Society of Gynaecology and Obstetrics (SEGO) [10]; this means that after diagnosis by imaging and core-needle or vacuum-assisted biopsy, and the assessment of local spread by magnetic resonance imaging, cases are presented to the breast cancer committee which then recommends the type of procedure to be offered: breast-conserving surgery or mastectomy. Axillary lymph node involvement was assessed using imaging techniques (axillary ultrasound) and sentinel lymph node biopsy. The sentinel lymph node was analysed using one-step nucleic acid amplification.

After surgery, our centre's breast cancer committee recommends adjuvant radiotherapy, adjuvant chemotherapy or both, depending on certain risk factors (tumour stage, prognostic factors, molecular subtype, patient age, menopausal status, etc.). Further, women that are positive for oestrogen receptors are given hormone therapy.

\section{Data collection}

We retrospectively searched for all women undergoing breast cancer surgery within the established study period and classified them into two groups: group A composed of women referred from the BCEDP, after a routine mammogram; and group B composed of women referred from primary care or from other gynaecological clinics in our health region, with a diagnosis of suspected breast cancer. We considered interval cancer those being diagnosed in the age 50 to 69 years outside of the BCEDP.

We collected the following data in the case report form: age, type of tumour (IDC or DCIS, regardless of the histological type); breast cancer stage; type of surgery (breast-conserving surgery or mastectomy); detection of sentinel lymph node and histopathological status; axillary lymphadenectomy; and need for subsequent adjuvant therapy and type. A specific spreadsheet was created for data collection.

\section{Statistical analysis}

Categorical variables were described using absolute and relative frequencies. Continuous variables were described using mean and standard deviation (SD). For comparing groups, parametric tests (Student's t-tests) were used for quantitative variables and chi-square tests for qualitative variables. Statistical analysis was performed with the IBM SPSS Statistics for Windows, Version 22 (Armonk, NY).

\section{Results}

In the study period, 630 surgical interventions were performed in our gynaecology department on 630 women diagnosed with breast cancer. The mean age of the women was 60.2 years (SD 5.4 years), 140 being under 50 years old $(22.2 \%)$ and 36 women under 40 years of age $(5.7 \%)$ at the time of surgery.

Out of the 630 women identified, 220 (35\%) were referred from the BCEDP and hence allocated to group A, while the others $(n=410,65 \%)$ were referred from primary care or their gynaecologists (group B). Overall, $329(52.2 \%)$ were between 50 and 69 years old. Of these, 200
(60.8\%) were diagnosed with breast cancer within the BCPEP and 129 (39.2\%) were diagnosed outside this programme (103 being interval cancers and 26 women who did not participate in the programme).

All women in group A were diagnosed by the mammography of the BCEDP while in group B 265 women (64.6\%) consulted due to selfpalpation of a breast nodule, having had blood secretion, experiencing breast pain, etc and 145 were diagnosed during a mammographic study performed out of the BCEDP. 92 out of the 103 interval cancers (89.3\%) in group B were invasive compared with $170(85 \%)$ in group $\mathrm{A}(\mathrm{p}=0.175)$.

Out of the 630 cases of breast cancer, 555 (88\%) were IDC and 75 (12\%) were DCIS. Among the women in group A, 185 were diagnosed with IDC ( $84 \%$ of all the cases in this group) and 35 with DCIS (16\%), while among the women in group B, 370 (90.2\%) had IDC and 40 DCIS $(9.8 \%)$ at diagnosis. There difference between groups in the number of cases of IDC was significant $(p=0.02)$. Table 1 shows the cases of breast cancer classified as a function of stage and patient group and Table 2 the cases of breast cancer classified as a function of patients age and patient group. Women were initially treated with neoadjuvant chemotherapy in 10 out of 220 cases (4.5\%) in group A and 77 out of 410 cases $(18.8 \%)$ in group $B(p<0.001)$. Mastectomies were performed in $47(21.4 \%)$ and $225(54.9 \%)$ women in groups A and B, respectively $(\mathrm{p}<0.001)$.

Sentinel lymph node detection was performed in 451 women: 159 and 292 in groups A and B, respectively. Sentinel lymph node involvement was found in $17 \%$ and $26.4 \%$ of women in groups $\mathrm{A}$ and $B$, respectively $(p<0.001)$, with axillary lymphadenectomies being performed in $31(14.1 \%)$ and $83(20.2 \%)$ cases in groups A and B, respectively $(\mathrm{p}=0.02)$. Adjuvant radiotherapy was given to $94 \%$ and $83.2 \%$ and adjuvant chemotherapy to $30 \%$ and $36.1 \%$ of women in groups $A$ and $B$, respectively ( $\mathrm{p}=0.469$ and $\mathrm{p}<0.001$ ) (Tables 2 and 3 ).

\begin{tabular}{|c|c|c|}
\hline Stage & Group A & Group B \\
\hline 0 & $13 / 220(5.9 \%)$ & $11 / 410(2.7 \%)$ \\
\hline I & $115 / 220(52.3 \%)$ & $212 / 410(29.5 \%)$ \\
\hline II & $72 / 220(32.7 \%)$ & $125 / 410(30.5 \%)$ \\
\hline II & $9 / 220(4 \%)$ & $69 / 410(16.8 \%)$ \\
\hline
\end{tabular}

Table 1: Cases of breast cancer classified by stage and patient group.

\begin{tabular}{|c|c|c|}
\hline Group of age & Group A & Group B \\
\hline$<30$ years & -- & $3 / 410(0.7 \%)$ \\
\hline $30-39$ years & -- & $33 / 410(8 \%)$ \\
\hline $40-49$ years & $13 / 220(5.9 \%)$ & $91 / 410(22.2 \%)$ \\
\hline $50-59$ years & $101 / 220(45.9 \%)$ & $77 / 410(18.8 \%)$ \\
\hline $60-69$ years & $106 / 220(48.2 \%)$ & $52 / 410(12.7 \%)$ \\
\hline $70-79$ years & -- & $77 / 410(18.8 \%)$ \\
\hline $80-90$ years & -- & $77 / 410(18.8 \%)$ \\
\hline
\end{tabular}

Table 2: Cases of breast cancer classified by age of patients and patient group.

\begin{tabular}{|c|c|c|c|c|c|}
\hline \multirow{2}{*}{ Distribution } & \multicolumn{2}{|c|}{ Group A } & \multicolumn{2}{c|}{ Group B } & \multirow{2}{*}{ P-value } \\
\cline { 2 - 5 } & $\mathbf{N}$ & $\mathbf{( \% )}$ & $\mathbf{N}$ & $\mathbf{( \% )}$ & \\
\hline Invasive ductal carcinoma & 185 & 84 & 370 & 90.2 & 0.02 \\
\hline Neoadjuvant chemotherapy & 10 & 4.5 & 77 & 18.8 & $<0.001$ \\
\hline Breast-conserving surgery & 173 & 78.6 & 185 & 45.1 & $<0.001$ \\
\hline Positive sentinel lymph node & 27 & 17 & 77 & 26.4 & $<0.001$ \\
\hline Axillary lymphadenectomy & 31 & 14.1 & 83 & 34.3 & 0.02 \\
\hline Adjuvant radiotherapy & 207 & 97.6 & 345 & 96.1 & 0.469 \\
\hline Adjuvant chemotherapy & 66 & 30 & 148 & 36.1 & $<0.001$ \\
\hline
\end{tabular}

Table 3: Distribution of the study variables by group (Group A: women referred from the screening programme; group $B$ : women referred from primary care or their gynaecologist). 


\section{Discussion}

Our study highlights that breast cancer diagnoses through our screening programme account for just over a third (35\%) of all the surgical interventions associated with this condition. Although this percentage may seem low, we should consider that the BCEDP includes women between 50 and 69 years old, and that in our series, 301 patients (47.8\%) were outside this age range, $140(22.2 \%)$ being under 50 years of age and $161(25.5 \%)$ over 69 years of age at diagnosis. It seems clear that the BCEDP is beneficial for certain age groups but does not have a significant impact on diagnoses in women outside the screening age range in our setting. Despite this, our results indicate that women referred through the BCEDP are diagnosed with less invasive breast cancer than those diagnosed outside the programme; they require less neoadjuvant chemotherapy, are more likely to have breast-conserving surgery and require less adjuvant chemotherapy.

In general, it is considered that women whose cancer is detected through screening programmes have a better prognosis than women who opt out of screening or who are diagnosed outside the age range established for screening [11]. Women being diagnosed with earlier stage and less invasive tumours may explain why BCEDP participants receive less aggressive surgery $(78.6 \%$ of interventions being breastconserving) and need less neoadjuvant chemotherapy. Our results are in line with those of the Norwegian screening programme in which the rate of breast-conserving surgery was twice as high in participants compared to non-participants in their programme $(334.4 / 100,000 \mathrm{vs}$. $167.6 / 100,000)$ [9].

One of the criticisms of breast cancer screening programmes is that they may lead to an over diagnosis of DCIS, which is associated with a low risk of evolving to invasive cancer and unlikely to becoming life threatening [12]. In our experience, we also found more diagnoses of DCIS or early-stage cancer in the screening programme participants than non-participants (16\% vs 9.6\%). These data agree with those of large American series that report that $20 \%$ of new cases of breast cancer diagnosed every year correspond to stage 0 [13] and European-based research that has found DCIS detection rates of 10 to $30 \%$ [14].

Bleyer and Welch [15] using Surveillance Epidemiology and End Results (SEER) data examined the trends from 1976 through 2008 in the incidence of early-stage breast cancer and large-stage breast cancer among women 40 years of age or older. They found the introduction of screening mammography in the United States has been associated with a doubling in the number of cases of early-stage breast cancer (from 112 to 234 cases per 100.000 women) and a decrease in the number of cases of large-stage breast cancer (from 102 to 94 cases per 100.000 women). They conclude there is a substantial overdiagnosis, accounting for nearly a third of all newly diagnosed breast cancers.

In line with international recommendations [16], our protocol includes sentinel lymph node biopsy in most cases. In our series, 451 out of 630 patients (71.6\%) underwent sentinel lymph node biopsy. The difference in metastatic involvement of the sentinel lymph node between the groups (17\% and $26.4 \%$ in group A and B, respectively) was not significant, and our rate of sentinel lymph node metastasis was like that found in other research [17]. In contrast, we did observe a difference in the rate of axillary lymphadenectomy between the groups, with a lower rate in women in group A $(14.1 \%$ vs $20.2 \%$ in group B; $\mathrm{p}=0.02)$. Having analysed sentinel lymph node involvement using onestep nucleic acid amplification, axillary lymphadenectomy was carried out in those with an mRNA copy number of over 15,000. The results confirm less metastatic lymph node involvement among women in group A than those in group B, this translating to less need for axillary lymphadenectomy.
In our series, a strikingly high number of patients had interval cancer, defined as cases of breast cancer diagnosed in the period between two programmed mammograms under the screening programme. In our experience, almost a third (31.3\%) of cases of breast cancer diagnosed among 50 to 69 -year-old women corresponded to interval cancer. A similar rate of interval cancer was reported by Tabar et al. 30 years ago [18]. In their study, based on databases of the screening programme in two Swedish counties, they found a rate of interval cancer of as high as $40 \%$ when mammograms were performed every other year. In analysis of the subgroup of 50 to 69 -year-old women, comparing data on cases of interval cancer and those of cancer diagnosed within the screening programme, we can conclude that the differences are like those found when overall comparisons are made between groups $A$ and $B$ in our study.

In our centre, $87 \%$ of the women who underwent surgery for breast cancer received adjuvant radiotherapy. We did not find significant differences between the study groups. This is attributable to the fact that radiotherapy after breast-conserving surgery reduces the risk of local recurrence [19] and, in our case, all women that undergo breastconserving surgery are treated with radiotherapy.

Our study has certain limitations. First, it was not designed and is not able to assess the impact of the screening programme on the breast cancer-associated mortality. It has been found that this type of secondary prevention programmes is able to reduce breast cancerassociated mortality by up to $21 \%$ in women over 50 years of age [20]. Similarly, we cannot assess the impact of false positives given that our study focused on histologically-confirmed cases that were treated surgically.

The main strength of our study is that includes all the cases of breast cancer that occurred in our health region, given that our hospital is the referral centre for this type of disease and, in our region, breast cancer is exclusively treated by gynaecologists of our breast care unit. Hence, the study reflects reality and is representative of what is happening among women in our setting.

\section{Ethical Considerations}

Given that this was a retrospective study analysing medical reports of patients already treated and that data collection was anonymous, the current Spanish legislation did not require informed consent to be obtained from the patients. On the other hand, the study was approved by the clinical research ethics committee of our hospital.

\section{Funding}

This study was funded through our department using a grant received from the Alava Association for Gynaecological and Obstetric Research (APIGOA).

\section{Conclusion}

In our setting, breast cancer patients who are referred from the BCEDP have earlier stage cancer, can be expected to benefit from less radical surgical procedures, have lower proportion of axillary lymph node involvement and require less complementary adjuvant chemotherapy.

\section{Acknowledgements}

We would like to thank Arantza Saez De Lafuente Morínigo from the Statistical Methodology Unit of Araba Integrated Healthcare Organisation for her help interpreting the results. 
Citation: Lete I, Aguiar M, Andonegui A, Zeberio N, Cuadra M et al. (2018) Impact of a Screening Program in the Diagnosis, Surgical Treatment and Management of Breast Cancer. J Cancer Sci Ther 10: 152-154. doi: 10.4172/1948-5956.1000537

\section{References}

1. Tabár L, Fagerberg CJ, Gad A, Baldetorp L, Holmberg LH, et al. (1985) Reduction in mortality from breast cancer after mass screening with mammography: Randomised trial from the breast cancer screening working group of the Swedish National Board of Health and Welfare. Lancet 325: 829832.

2. Myers ER, Moorman P, Gierisch JM, Havrilesky LJ, Grimm LJ, et al. (2015) Benefits and harms of breast cancer screening: A systematic review. JAMA 314: 1615-1634.

3. Barratt A (2015) Overdiagnosis in mammography screening: A 45-year journey from shadowy idea to acknowledged reality. BMJ p: 350.

4. Broeders MJM, Ripping TM, Hubbard RA (2016) Weighing the benefits and harms: Screening mam-mography in the balance. Breast Cancer Screening pp: 51-58.

5. Haukka J, Byrnes G, Boniol M, Autier P (2011) Trends in breast cancer mortality in Sweden before and after implementation of mammography screening. PLoS ONE 6: e22422.

6. Gotzsche PC, Jorgensen KJ (2013) Screening for breast cancer with mammography. Cochrane Database Syst Rev 6: CD001877.

7. Independent UK Panel on Breast Cancer Screening (2012) The benefits and harms of breast cancer screening: An independent review. Lancet 380: 1778-1786.

8. Broeders M, Moss S, Nystrom L, Njor S, Jonsson H, et al. (2012) The impact of mammographic screening on breast cancer mortality in Europe: A review of observational studies. J Med Screen 19: 14-25.

9. Hofvind S, Lee Cl, Elmore JG (2012) Stage-specific breast cancer incidence rates among participants and non-participants of a population-based mammographic screening program. Breast Cancer Res Treat 135: 291-299.

10. Ongoguía SEGO (2017) Infiltrating breast cancer 2017. Clinical practice guides in gynecological and breast cancer. SEGO publications.
11. Tabar L, Fagerberg G, Duffy SW, Day NE, Gad A, et al. (5372) Update of the Swedish two-county program of mammographic screening for breast cancer. Radiol Clin North Am 30: 187-210.

12. Brennan M, Houssami N (2016) Discussing the benefits and harms of screening mammography. Maturitas 92: 150-153.

13. Swain M, Jeudy M, Pearlman MD (2016) Controversies in screening mammography. Clin Obstet Gynecol 59: 351-361.

14. Zackrisson S, Andersson I, Janzon L, Manjer J, Garne JP (2006) Rate of over-diagnosis of breast cancer 15 years after end of Malmo mammographic screening trial: Follow-up study. BMJ 332: 689-692.

15. Bleyer A, Welch HG (2012) Effect of three decades of screening mammography on breast-cancer incidence. New Engl J Med 367: 5378-2005.

16. Giuliano AE, Hunt KK, Ballman KV, Beitsch PD, Whitworth PW, et al. (2011) Axillary dissection $v s$ no axillary dissection in women with invasive breast cancer and sentinel node metastasis: A randomized clinical trial. JAMA 305: 569-575.

17. Giuliano AE, Kirgan DM, Guenther JM, Morton DL (5374) Lymphatic mapping and sentinel lymphadenectomy for breast cancer. Annals Surg 220: 391-401.

18. Tabar L, Faberberg G, Day NE, Holmberg L (1987) What is the optimum interval between mammographic screening examinations? An analysis based on the latest results of the Swedish two-county breast cancer screening trial. $\mathrm{Br} \mathrm{J}$ Cancer 55: 547-551.

19. Van Maaren MC, De Munck L, De Bock GH, Jobsen JJ, Van Dalen T, et al. (2016) 10 year survival after breast-conserving surgery plus radiotherapy compared with mastectomy in early breast cancer in the Netherlands: A population-based study. Lancet Oncol 17: 1158-1170.

20. Nystrom L, Andersson I, Bjurstam N, Frisell J, Nordenskjöld B, et al. (2002) Long-term effects of mammography screening: Updated overview of the Swedish randomised trials. Lancet 359: 909-919. 